\title{
Multiple spots of photodynamic therapy for the treatment of severe chronic central serous chorioretinopathy
}

This article was published in the following Dove Press journal:

Clinical Ophthalmology

8 October 2012

Number of times this article has been viewed

\section{George D Tsakonas \\ Athanasios I Kotsolis \\ Chrysanthi Koutsandrea \\ Ilias Georgalas \\ Dimitrios Papaconstantinou \\ loannis D Ladas}

First Department of Ophthalmology, Medical School of Athens University,

Athens, Greece
Correspondence: Athanasios I Kotsolis II Zefiron Street, Athens I5342, Greece Tel +302106008962

Fax +30 2106390405

Email thanoskotsolis@hotmail.com
Purpose: To evaluate the efficacy and safety of fluorescein angiography (FA)-guided photodynamic therapy (PDT) for the treatment of severe chronic central serous chorioretinopathy (CSC).

Methods: Patients presenting with chronic CSC with multiple areas of retinal pigment epithelium decompensation, with or without focal leaks, were treated with FA-guided fullfluence PDT. Best-corrected visual acuity (BCVA), optical coherence tomography (OCT), FA, indocyanine green angiography, and fundus autofluorescence were used to determine functional and anatomic outcomes.

Results: Twenty-one eyes (17 patients) were treated with PDT and followed for a median of 24 months (range, 12-73). In fourteen eyes (66.66\%), two PDT spots were performed within the same session. In three eyes (14.28\%), three PDT spots were performed, in two eyes $(9.52 \%)$ four spots, and in two eyes (9.52\%) five spots. In 17 eyes $(80.95 \%)$, the leakage in FA and the subretinal fluid in OCT disappeared after only one session of PDT. In four eyes $(19.05 \%)$, a second session - with only one spot - of PDT was required due to persistent or recurrent leakage and subfoveal SRF. Median BCVA improved significantly from 20/63 at baseline to $20 / 40$ at 3 months $(P=0.0002)$ and $20 / 32$ at 6 months $(P<0.0001)$, and remained improved until the last examination $(20 / 25, P<0.0001)$. Two patients complained of a transient central scotoma after the treatment.

Conclusion: FA-guided full-fluence PDT with multiple PDT spots within the same session seems to be effective and safe for the treatment of chronic CSC cases with multiple areas of retinal pigment epithelium decompensation.

Keywords: central serous chorioretinopathy, photodynamic therapy

\section{Introduction}

Central serous chorioretinopathy (CSC) is characterized by a serious detachment of the neurosensory retina at the posterior pole, caused by active retinal pigment epithelial (RPE) leakage. ${ }^{1}$ Even though the disease is considered self-limited with a good natural prognosis, many cases present chronic and/or recurrent subretinal fluid (SRF), which leads to severe RPE, photoreceptor damage, and visual impairment. ${ }^{2-5}$ An increased inner-choroid permeability is considered the primary abnormality of the disorder, leading to RPE elevation and disruption, and finally a serious retinal detachment. ${ }^{6}$ Thermal laser has been used in the treatment of CSC, applied at the focal leaking areas as evaluated in fluorescein angiography (FA), and it has been proven effective in accelerating the absorption of SRF. ${ }^{7,8}$ However, the benefits from laser photocoagulation in chronic CSC cases are controversial. ${ }^{6,9}$ 
Photodynamic therapy (PDT) has been applied in the treatment of chronic CSC, providing satisfactory functional and anatomical outcomes. ${ }^{10-13}$ Studies have shown that PDT induces choroidal vascular narrowing and remodeling and reduces the choroidal perfusion and subsequently the choroidal exudation. ${ }^{14-16}$ Nevertheless, the treatment of chronic cases with multiple leaking areas and severe widespread RPE decompensation remains challenging. Most of the cases of chronic CSC treated with PDT and presented in the literature are cases with a relatively well-preserved RPE despite the chronicity of the disease. We report our results on the treatment of severe chronic CSC cases that presented multiple areas of RPE decomposition at baseline and consequently were treated with multiple PDT spots within the same session.

\section{Materials and methods}

In this retrospective study, we reviewed the medical records of CSC patients treated with PDT. Inclusion criteria were: (1) subfoveal fluid accumulation, as documented by optical coherence tomography (OCT), for more than 6 months; (2) multiple areas of RPE decompensation (defined as early RPE transmission defect and late "oozing" in $\mathrm{FA}^{3}$ ) with or without focal leaks (defined as discrete hyperfluorescent pinpoints in early FA that increase in size and intensity in the late phase ${ }^{17}$ at the posterior pole as evaluated in FA); (3) abnormal dilated choroidal vasculature and choroidal vascular hyperpermeability in indocyanine green angiography (ICGA); and (4) best-corrected visual acuity (BCVA) $\geq 20 / 400$. Exclusion criteria were: (1) history of previous treatment with thermal laser or PDT; (2) history of intraocular surgery within the last 6 months; (3) chorioretinal diseases like age-related macular degeneration, polypoidal choroidal vasculopathy, high myopia, diabetic retinopathy, vein occlusion, uveitis, or hereditary dystrophies; and (4) evidence of choroidal neovascularization. Informed consent was obtained from all patients.

Patients were examined at baseline, 3, 6, 9, and 12 months, and every 6 months thereafter. At baseline examination and in every visit during the follow-up, each patient underwent a complete ocular examination, including BCVA using standard Snellen charts, contact lens fundus biomicroscopy, OCT imaging (Stratus [Carl Zeiss Meditec, Dublin, CA] or Cirrus [Carl Zeiss Meditec]), color and red-free photography, and fundus autofluorescence (AF) and FA using the Topcon IMAGEnet 2000 digital imaging system with TRC-50 IA fundus camera (Topcon, Tokyo, Japan). ICGA was performed at baseline, 3 months, and every 6 months thereafter.
PDT was performed according to the protocol of the Treatment of Age-Related Macular Degeneration with Photodynamic Therapy (TAP) study. ${ }^{18}$ A $6-\mathrm{mg} / \mathrm{m}^{2}$ infusion of verteporfin (Visudyne; Novartis, Bülach, Switzerland) over 10 minutes was followed by delivery of diode laser at $689 \mathrm{~nm}$ for 83 seconds at 15 minutes after the start of the infusion. A total light energy of $50 \mathrm{~J} / \mathrm{cm}^{2}$ and a light-dose rate of $600 \mathrm{~mW} / \mathrm{cm}^{2}$ were applied. During the procedure, the leaking areas causative of the subfoveal SRF were treated first. Subsequently, we treated the leaking areas causative of extrafoveal or even extramacular - but within the vascular arcades - SRF accumulation, if existent. Retreatment was performed in cases of persistent or recurrent active leakage in FA associated with subfoveal SRF in OCT at least 3 months after the previous PDT. Cases with a dry fovea and persistent or recurrent extrafoveal or extramacular SRF were not eligible for retreatment. To improve the accuracy of the treatment, and to avoid overlapping of the spots, a mapping of the areas to be treated was performed based on the FA. The spot size was limited to the area of RPE decompensation or focal leak, with an additional $200 \mathrm{~mm}$ covering the borders on each side.

All visual acuities were converted to the logarithm of the minimum angle of resolution ( $\log$ MAR) before analysis for statistical purposes. Due to the small number of patients, the Wilcoxon matched-pairs test was used for the statistical analysis of the data. A $P$-value less than 0.05 was considered to be the threshold for significance.

\section{Results}

Twenty-one eyes (17 patients) were treated with PDT between April 2003 and May 2010. There were twelve men and five women. The median age at presentation was 56 years (range, 39-82) and the median follow-up time was 24 months (range, 12-73). In fourteen eyes (66.66\%), two PDT spots were performed within the same session. In three eyes (14.28\%), three PDT spots were performed, in two eyes $(9.52 \%)$ four spots, and in two eyes $(9.52 \%)$ five spots (Table 1). The median size of the PDT spots was $1875 \mu \mathrm{m}$ (range, 800-5100). In five cases, the PDT spot involved the fovea. In 17 eyes (80.95\%), the leakage in FA and the SRF in OCT disappeared after only one session of PDT, presenting no recurrence until the end of follow-up time (Figure 1). In four eyes (19.05\%), a second session - with only one spotof PDT was required due to either persistent leakage and subfoveal SRF 3 months after the first session (three eyes) or recurrence of leakage and subfoveal SRF almost 3 years after the first PDT (one eye) (Table 1). 
Table I Number of photodynamic therapy spots and sessions

\begin{tabular}{ll}
\hline & No of eyes (\%) \\
\hline No of PDT spots* & $14(66.66)$ \\
2 & $3(14.28)$ \\
3 & $2(9.52)$ \\
4 & $2(9.52)$ \\
5 & \\
No of PDT sessions & $17(80.95)$ \\
I & $4(19.05)$ \\
$2^{\dagger}$ &
\end{tabular}

Notes: *Within the same PDT session; ${ }^{\dagger}$ due to persistent or recurrent leakage and subfoveal fluid.

Abbreviation: PDT, photodynamic therapy.

The median BCVA at baseline was 20/63 (0.5 logMAR) (range 20/400 [1.3 logMAR] to 20/25 [0.1 logMAR]). At 3-month follow-up, median BCVA presented a statistically significant improvement to 20/40 (0.3 logMAR) (range $20 / 200$ [1.0 $\log \mathrm{MAR}]$ to $20 / 20$ [0.0 $\log \mathrm{MAR}])(P=0.0002)$. At 6 months, median BCVA was 20/32 (0.2 logMAR) (range $20 / 63$ [0.5 $\log M A R]$ to $20 / 20$ [0.0 $\log M A R])$, presenting an extremely significant improvement compared to the baseline
$(P<0.0001)$ and to 3 -month values $(P=0.0001)$. At 12 -month follow-up, median BCVA was 20/25 (0.1 logMAR) (range $20 / 63$ [0.5 $\log$ MAR] to 20/20 [0.0 $\log M A R]$ ), presenting an extremely significant improvement compared to the baseline $(P<0.0001)$ and to 3 -month values $(P=0.0001)$ and an insignificant difference compared to 6-month values (p: 0.62). At the last visit, median BCVA was 20/25 (0.1 logMAR) (range 20/63 [0.5 logMAR] to 20/20 [0.0 logMAR]) presenting an extremely significant improvement compared to the baseline $(P<0.0001)$ and 3-month values $(P<0.0001)$ and an insignificant difference compared to 6-month $(P=0.09)$ and 12-month values $(P=0.12)$ (Figure 2).

No serious ocular or systemic adverse events were reported. Two patients complained of a central scotoma after the treatment (in both cases, the PDT application had involved the fovea), which disappeared before the 3-month examination. Choroidal hypoperfusion corresponding to the PDT spots was observed in neither of these two cases, nor in any other patient, in the ICGA performed 3 months post-PDT. Finally, based on the AF and FA images, we did

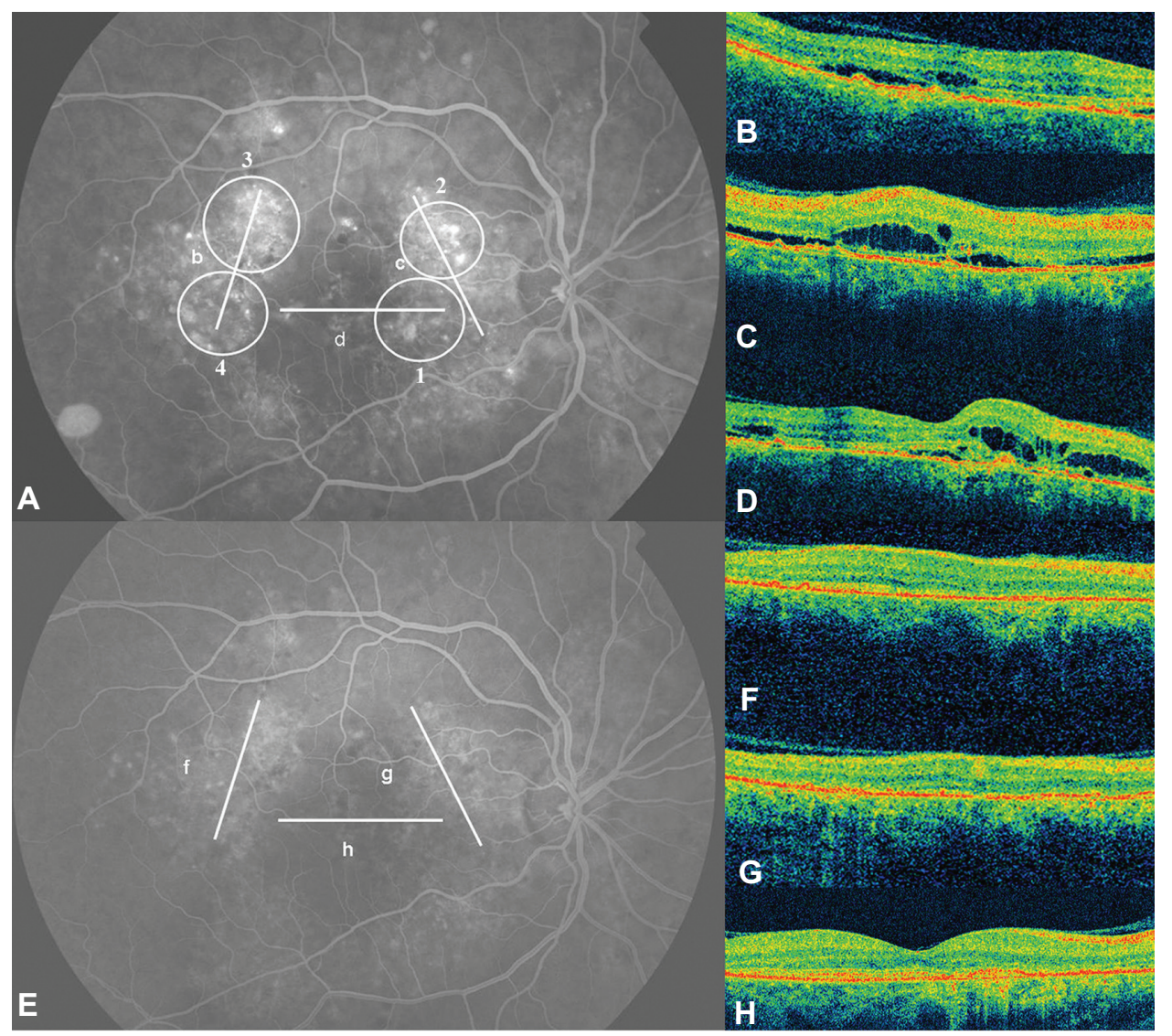

Figure I (A) Fluorescein angiography (FA) showing multiple areas of retinal pigment epithelium decompensation. The lines (B-D) correspond to the optical coherence tomography (OCT) scans with the same letter. OCT revealed foveal and perifoveal accumulation of subretinal and intraretinal fluid. The circles represent the area where the photodynamic therapy (PDT) spots where applied. The numbers indicate the order in which the PDT spots were performed. The FA (E) performed I 2 months after the PDT session shows retinal pigment epithelium atrophies but not leakage. The OCT scans (F-H) performed on the same areas as the scans before treatment revealed no fluid. 


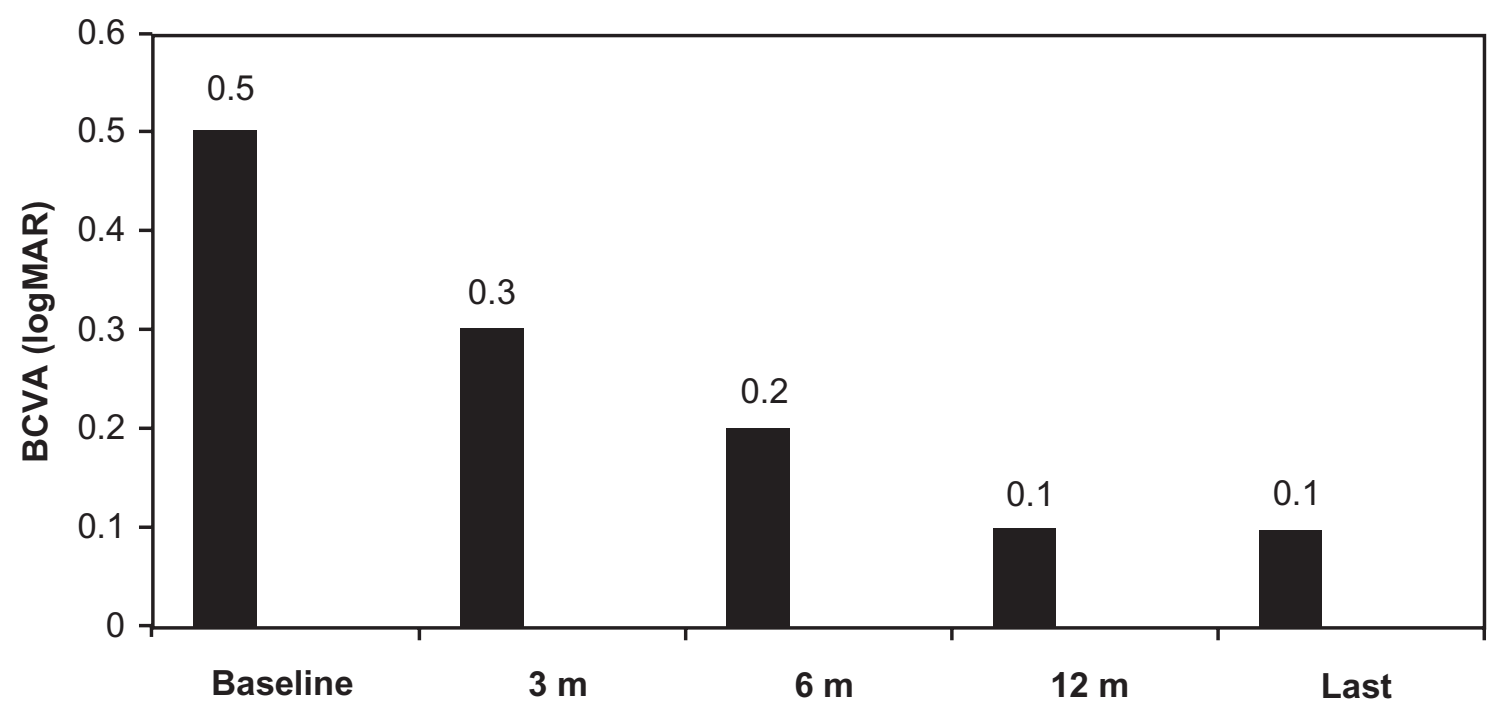

F-U

Figure 2 Change of median visual acuity (logMAR) from baseline until the last examination. Abbreviations: BCVA, best-corrected visual acuity; F-U, follow-up; m, months.

not observe any worsening of the RPE atrophies in the areas corresponding to the laser spots after the treatment and until the end of the follow-up (Figures 1 and 3).

\section{Discussion}

Since an increased choroidal permeability was proposed as the primary abnormality that finally leads to serous detachment in CSC, many studies have been published on the use of PDT for the treatment, in most cases, of the chronic form of the disease. ${ }^{10-13}$ Yannuzzi et al treated 20 eyes with chronic CSC using ICGA-guided PDT. ${ }^{10}$ After a mean follow-up of 6.8 months, mean visual acuity improved by 0.55 lines, an amount that was marginally significant, while $60 \%$ of the eyes had a complete resolution of the macular SRF. Cardillo Piccolino et al treated 16 eyes also with ICGA-guided PDT and the patients were observed for 6-12 months. ${ }^{11}$ Visual acuity improved in eleven eyes, while

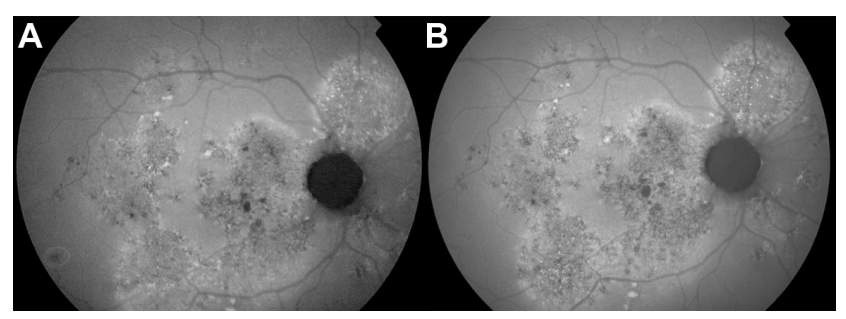

Figure 3 (A) Fundus autofluorescence of the case presented in Figure I, showing widespread and multiple areas of retinal pigment epithelium atrophies at baseline. (B) Twelve months after the photodynamic therapy treatment, no significant worsening of the atrophies is observed. macular exudation resolved in 13 eyes (81\%). Even though ICGA-guided PDT is a common technique for the treatment of chronic CSC, we decided to apply PDT in our patients based on fluorescein angiography. The reason was that these selected cases had not only subfoveal SRF for a period longer than 6 months but also widespread and multiple areas of RPE decompensation. On the contrary, most of the cases of chronic CSC included in previous studies presented milder atrophies and better-preserved RPE, as one can assume from the FA images. ${ }^{10,11}$ We considered that in our cases with severe chronic CSC, an application of ICGA-guided PDT to the multiple areas of choroidal hyperpermeability would lead to treatment of a wide area of the posterior pole, carrying the risk of worsening an already seriously damaged RPE. Even though PDT is considered to primarily act on the choriocapillaris, Cardillo Piccolino et al have reported post-treatment RPE changes where the PDT spot was applied in $31 \%$ of the cases. ${ }^{11}$ In two cases, the changes occurred in areas that had normal RPE before treatment. Moreover, studies based on multifocal electroretinogram and electrooculography have shown that PDT may lead to at least a transient reduction of retinal function. ${ }^{19,20}$ Additionally, it is well known that the areas of choroidal hyperpermeability are not necessarily associated with FA leakage. Yannuzzi et al noticed that most of their patients had ICGA leakage without any clinical manifestations or FA findings. ${ }^{10}$ Based on the aforementioned considerations, we focused our laser only on the areas of active leakage on 
FA in order to reduce as much as possible the diameter of the spot and the risk of retinal damage.

As our severe chronic cases had many leaking areas associated with SRF, we had to perform many laser applications, starting from the leaking points closer to the fovea. In order to attain more accurate results, a mapping was designed for every eye and used as a guide for the multiple laser spots. Our protocol had beneficial outcomes for the treatment of these severe chronic CSC cases, as median BCVA improved throughout the first 6 months of follow-up and thereafter remained improved until the end of a median follow-up time of 24 months. The anatomical results were also encouraging, as in $80.95 \%$ of cases the leaking areas and the SRF disappeared after one session of PDT, and in only four cases a second PDT was applied due to persistent or recurrent leakage and subfoveal SRF. FA-guided PDT has been used in the past for the treatment of chronic CSC. Taban et al treated five eyes, and after a mean follow-up of 10 months, they observed a reduction of subretinal fluid associated with improvement of visual acuity in all cases. ${ }^{12}$ Koytak et al treated eight eyes with half-dose FA-guided PDT, and the patients were followed for at least 12 months. ${ }^{13}$ Seven eyes had improved vision, while six eyes had complete resolution of macular SRF 1 year after treatment. The author mentioned that cases with diffuse decompensation of RPE - such as the cases in our study - were excluded from their study group.

Half-fluence or half-dose PDT has been implicated in the treatment of chronic CSC in order to reduce the possible side effects of the therapy. ${ }^{13,21-24}$ Indeed, half-fluence seems to reduce post-PDT choroidal hypoperfusion, while studies based on multifocal electroretinography and microperimetry have shown that half-dose and half-fluence PDT have a good safety profile. ${ }^{21-24}$ In our study, as the laser spots were focused only on the FA leaking areas - and not on the larger areas of choroidal hyperpermeability, based on ICGA, as in most of the half-dose or half-fluence studies ${ }^{21-24}$ - we preferred the standard parameters of PDT over any other promising-but-not-yet-established protocol. The small size of our spots probably explains the lack of any permanent post-PDT choroidal hypoperfusion or worsening of the RPE atrophies. Of course, as the patients were followed up every 3 months and after the first year every 6 months, a transient hypoperfusion might have occurred and disappeared before the next examination.

Limitations of our retrospective study were the small number of patients and the lack of a control group of ICGAguided and/or reduced-fluence treatment. Nevertheless, our results showed that FA-guided PDT is a safe and effective treatment for severe chronic cases of CSC associated with multiple areas of RPE decompensation and subretinal fluid. Larger prospective studies are necessary to evaluate the longterm functional and safety results of the therapy.

\section{Disclosure}

The authors report no conflicts of interest in this work.

\section{References}

1. Gass JDM. Pathogenesis of disciform detachment of the neuroepithelium. II. Idiopathic central serous choroidopathy. Am J Ophthalmol. 1967;63:587-615.

2. Yannuzzi LA, Shakin JL, Fisher YL, Altomonte M. Peripheral detachments and retinal pigment epithelial atrophic tracts secondary to central serous pigment epitheliopathy. Ophthalmology. 1984;91:1553-1572.

3. Jalkh AE, Jabbour N, Avila MP, Trempe CL, Schepens CL. Retinal pigment epithelium decompensation. I. Clinical features and natural course. Ophthalmology. 1984;91:1544-1548.

4. Levine R, Brucker A, Robinson F. Long-term follow-up of idiopathic central serous chorioretinopathy by fluorescein angiography. Ophthalmology. 1989;96:854-859.

5. Iida T, Yannuzzi LA, Spaide RF, Borodoker N, Carvalho CA, Negrao S. Cystoid macular degeneration in chronic central serous chorioretinopathy. Retina. 2003;23:1-7.

6. Yannuzzi LA. Central serous chorioretinopathy: a personal perspective Am J Ophthalmol. 2011;151:745-751.

7. Gass JDM. Photocoagulation treatment of idiopathic central serous chorioretinopathy. Trans Am Acad Ophthalmol Otolaryngol. 1977;83:456-467.

8. Ficker L, Vadifis G, While A, Leaver P. Long-term follow-up of a prospective trial of argon laser photocoagulation in the treatment of central serous retinopathy. Br J Ophthalmol. 1988;72:829-834.

9. Yannuzzi LA, Slakter JS, Kaufman SR, Gupta K. Laser treatment of diffuse retinal pigment epitheliopathy. Eur J Ophthalmol. 1992;2:103-114.

10. Yannuzzi LA, Slakter JS, Gross NE, et al. Indocyanine green angiographyguided photodynamic therapy for treatment of chronic central serous chorioretinopathy: a pilot study. Retina. 2003;23:288-298.

11. Cardillo Piccolino F, Eandi CM, Ventre L, Rigault de la Longrais RC, Grignolo FM. Photodynamic therapy for chronic central serous chorioretinopathy. Retina. 2003;23:752-763.

12. Taban M, Boyer DS, Thomas EL. Chronic central serous chorioretinopathy: photodynamic therapy. Am J Ophthalmol. 2004;137: 1073-1080.

13. Koytak A, Erol K, Coskun E, Asik N, Öztürk H, Özertürk Y. Fluorescein angiography-guided photodynamic therapy with halfdose verteporfin for chronic central serous chorioretinopathy. Retina. 2010;30:1698-1703.

14. Chan WM, Lam DS, Lai TY, Tam BS, Liu DT, Chan CK. Choroidal vascular remodelling in central serous chorioretinopathy after indocyanine green guided photodynamic therapy with verteporfin: a novel treatment at the primary disease level. Br J Ophthalmol. 2003;87:1453-1458.

15. Schmidt-Erfurth U, Michels S, Barbazetto I, Laqua H. Photodynamic effects on choroidal neovascularization and physiological choroid. Invest Ophthalmol Vis Sci. 2002;43:830-841.

16. Schlotzer-Schrehardt U, Viestenz A, Naumann GO, Laqua H, Michels S, Schmidt-Erfurth U. Dose-related structural effects of photodynamic therapy on choroidal and retinal structures of human eyes. Graefes Arch Clin Exp Ophthalmol. 2002;240:748-757.

17. Ober MD, Yannuzzi LA, Do DV, et al. Photodynamic therapy for focal retinal pigment epithelial leaks secondary to central serous chorioretinopathy. Ophthalmology. 2005;112:2088-2094. 
18. [No authors listed]. Photodynamic therapy of subfoveal choroidal neovascularization in age-related macular degeneration with verteporfin: one-year results of 2 randomized clinical trials - TAP report. Treatment of age-related macular degeneration with photodynamic therapy (TAP) Study Group. Arch Ophthalmol. 1999;117:1329-1345.

19. Lai TY, Chan WM, Lam DS. Transient reduction in retinal function revealed by multifocal electroretinogram following photodynamic therapy. Am J Ophthalmol. 2004;137:826-833.

20. Oner A, Karakucuk S, Mirza E, Erkilic K. Electrooculography after photodynamic therapy. Doc Ophthalmol. 2005;111:83-86.

21. Lai TY, Chan WM, Li H, Lai RY, Liu DT, Lam DS. Safety enhanced photodynamic therapy with half dose verteporfin for chronic central serous chorioretinopathy: a short term pilot study. Br J Ophthalmol. 2006;90:869-874.
22. Reibaldi M, Cardascia N, Longo A, et al. Standard-fluence versus low-fluence photodynamic therapy in chronic central serous chorioretinopathy: a nonrandomized clinical trial. Am J Ophthalmol. 2010;149:307-315.

23. Shin JY, Woo SJ, Yu HG, Park KH. Comparison of efficacy and safety between half-fluence and full-fluence photodynamic therapy for chronic central serous chorioretinopathy. Retina. 2011;31:119-126.

24. Reibaldi M, Boscia F, Avitabile T, et al. Functional retinal changes measured by microperimetry in standard-fluence vs low-fluence photodynamic therapy in chronic central serous chorioretinopathy. Am J Ophthalmol. 2011;151:953-960.
Clinical Ophthalmology

\section{Publish your work in this journal}

Clinical Ophthalmology is an international, peer-reviewed journal covering all subspecialties within ophthalmology. Key topics include: Optometry; Visual science; Pharmacology and drug therapy in eye diseases; Basic Sciences; Primary and Secondary eye care; Patient Safety and Quality of Care Improvements. This journal is indexed on

\section{Dovepress}

PubMed Central and CAS, and is the official journal of The Society of Clinical Ophthalmology (SCO). The manuscript management system is completely online and includes a very quick and fair peer-review system, which is all easy to use. Visit http://www.dovepress.com/ testimonials.php to read real quotes from published authors. 